\title{
Ergodicity and Invariance of Flows in Queuing Systems
}

\author{
G. Sh. Tsitsiashvili \\ Institute for Applied mathematics, Far Eastern Branch of Russian Academy Sciences, Vladivostok, Russia \\ Email: guram@iam.dvo.ru
}

How to cite this paper: Tsitsiashvili, G.Sh (2018) Ergodicity and Invariance of Flows in Queuing Systems. Journal of Applied Mathematics and Physics, 6, 1454-1459. https://doi.org/10.4236/jamp.2018.67122

Received: June 27, 2018

Accepted: July 16, 2018

Published: July 19, 2018

Copyright (C) 2018 by author and Scientific Research Publishing Inc. This work is licensed under the Creative Commons Attribution International License (CC BY 4.0).

http://creativecommons.org/licenses/by/4.0/

\begin{abstract}
In this paper, we investigate the flow of customers through queuing systems with randomly varying intensities. The analysis of the Kolmogorov-Chapman system of stationary equations for this model showed that it is not possible to construct a convenient symbolic solution. In this paper an attempt is made to circumvent this requirement by referring to the ergodicity theorems, which gives the conditions for the existence of the limit distribution in the service processes, but do not require knowledge of them.
\end{abstract}

\section{Keywords}

A Queuing System, An Ergodicity, An Input Flow, A Randomly Varying Intensity

\section{Introduction}

In this paper, we investigate the flow of customers through queuing systems. This work was initiated by the task, which was set in 2000 to the author of the article by the late professor of the Australian National University (Canberra, Australia) Joe Gani. He proposed to calculate a model of a single-server queuing system with a Poisson input flow with a randomly changing intensity and a randomly changing service intensity. The analysis of the Kolmogorov-Chapman system of stationary equations for this model showed that it is not possible to construct a convenient symbolic solution and it is necessary to use numerical methods and to solve approximately a linear system of algebraic equations.

Progress in solving this problem could be obtained only recently, thanks to an appeal to Burke's theorem on the coincidence of Poisson input and output flow distributions in the $M|M| n \mid \infty$ system. However, the proof of this theorem [1] occurred to be rather cumbersome and inconvenient to extend to other queuing 
models, including systems in a random environment. Therefore, it was necessary to develop an alternative proof [2] of this theorem, based on the classical work of A.Ya. Khinchin [3] on the representation of Poisson flows.

However, this alternative proof required knowledge of limit distributions in queuing systems, which is not always possible to obtain in a symbolic form. Therefore, in this paper an attempt is made to circumvent this requirement by referring to the ergodicity theorems, which gives the conditions for the existence of the limit distribution in the service processes, but do not require knowledge of them.

\section{Equality of Average Intensities of Input and Output Flows in the Queuing System}

Consider queuing system $A$ with Poisson input flow of intensity $\lambda$. Let $x(t)$ be the number of customers of the input flow in the system $A$ on a half-interval $(0, t], x(0)=0$. Then the following relation is true.

lemma 1 . The following almost sure convergence is true:

$$
\frac{x(t)}{t} \rightarrow \lambda, t \rightarrow \infty .
$$

Proof. Let $[t]$ be an integer part of $t>1$. Then almost surely the following relations are executed:

$$
\frac{x([t])}{[t]} \cdot \frac{[t]}{[t]+1} \leq \frac{x(t)}{t} \leq \frac{x([t]+1)}{[t]+1} \cdot \frac{[t]+1}{[t]},
$$

with

$$
x([t])=\sum_{i=0}^{[t]-1}(x(i+1)-x(i)),
$$

where all terms in equality (3) are independent and have Poisson distribution with the parameter $\lambda$. Then from the relations (2), (3) and the strengthened law of large numbers (see, for example, ([4], Chapter $8, \$ 4 \$)$ ) we have the equality (1).

Corollary 1. From Lemma 1 we have the convergence by probability in the relation (1).

Denote $y(t)$ the number of customers that came out of the queuing system $A$ on the half-interval $(0, t]$ and put $z(t)$ the number of customers in the system at time $t$.

Theorem 1. If the random process $z(t)$ is ergodic and stationary, then the convergence by probability is true

$$
\frac{y(t)}{t} \rightarrow \lambda, t \rightarrow \infty
$$

Proof. With probability one, the following equality is performed

$$
x(t)=y(t)+z(t), t \geq 0 .
$$

In turn, from the ergodicity of the stationary random process $z(t), z(0)=0$ 
it follows (see, for example, ([4], Chapter $10, \$ 4 \$)$ ) that there is the distribution function $F(u), F(0)=0, F(u) \rightarrow 1, u \rightarrow \infty$ such that for any $u \geq 0$ the probability $P(z(t)<u) \equiv F(u)$. We prove now that the following convergence by probability is true

$$
\frac{z(t)}{t} \rightarrow 0, t \rightarrow \infty .
$$

Fix $\varepsilon>0$, then the following equality is true:

$$
P\left(\frac{z(t)}{t}>\varepsilon\right)=1-F(\varepsilon t) \rightarrow 0, t \rightarrow \infty,
$$

and so the convergence by probability in the relation (6) is valid. From the convergence by probability in the relation (6), equality (5) and Corollary 1 we obtain the statement of the theorem 1.

\section{Poisson Output Flows in Queuing Systems of General Form}

Suppose that the ergodic stationary process $z(t)$ is Markov. Since this process characterizes the number of customers in the queuing system $A$, then it takes the values $0,1, \cdots$ Denote $f_{k}=P(z(t)=k), k=0,1, \cdots$ and suppose that the intensities of $\mu_{k}$ customers leaving the system $A$, in the state $z(t)=k$.

Theorem 2. The output flow in queuing system $\mathrm{A}$ is Poisson with the intensity $\lambda$.

Proof. A random sequence of points on the real axis is called a Poisson flow with the intensity $\lambda$ if the following conditions ([3], page 12,13), ([5], page 20, 35-37) are true: 1) the probability of the existence of the point of flow on the time interval $[t, t+h)$ does not depend on the location of the points of the flow up to the time $t$ (this property is called lack of follow-through and expresses the mutual independence of the flow points into disjoint periods of time);2) the probability that in the half-interval $[t, t+h)$ the point of flow appears is $\lambda(t) h+o(h), h \rightarrow 0 ; 3)$ the probability of occurrence of two or more flow points in the half-interval $[t, t+h)$ is $o(h), h \rightarrow 0$.

We use the construction of [2] and prove that the output flow of customers from the queuing system $A$ is Poisson. Indeed, since the random process $z(t)$ is discrete Markov, and the moments of withdrawal of customers from the system $A$ are the moments of jumps down the process $z(t)$, the output flow of customers from $A$ satisfies the condition a), checking of the conditions b), c) is made by direct calculations. Therefore, the output flow in the system $A$ is Poisson with the intensity $a=\sum_{k=1}^{\infty} f_{k} \mu_{k}$. Using Theorem 1 , we obtain the equality $\lambda=a$.

Remark 1. In a case the queuing system A is n-server type $M|M| n \mid \infty$, Theorem 2 is a generalization of the famous theorem of Burke [1]. The peculiarity of this design is the fact, that only ergodic properties of the Markov process $z(t)$ are important, while knowledge of stationary probabilities 
$f_{k}, k=1,2, \cdots$ are not necessary to calculate the intensity of the output Poisson flow.

\section{Queuing Systems with Failures}

Consider the $M|M| 1 \mid 0$ queuing system, in which the customers coming on a busy server, is refused. Input flow to this system is a Poisson with the intensity $\lambda$, service times have exponential distribution with the parameter $\mu$. This system is described by a number $z(t)$ of the customers in it at the moment $t$ [5]. The set of states of the Markov process $z(t)$ is $\{0,1\}$. Denote transition intensity of the process $z(t)$ from state $i$ to state $j$ by $\alpha(i, j)$. Then the following equations are true:

$$
\alpha(0,1)=\lambda, \alpha(1,0)=\mu, \alpha(1,1)=\lambda .
$$

Here the transition $0 \rightarrow 1$ corresponds to the arrival of the customer into the empty system. In turn, the transitions between the process states $z(t)$, leading to the withdrawal of customers from the system have the form $1 \rightarrow 0,1 \rightarrow 1$ (see Figure 1).

The transition $1 \rightarrow 0$ corresponds to the withdrawal of the severed customer from the system, and the $1 \rightarrow 1$ corresponds to the withdrawal of the refused customer from the system.

Markov process $z(t)$ is ergodic for any parameter values $\lambda, \mu>0$, and its limit distribution has the form

$$
b_{0}=\frac{1}{1+\rho}, b_{1}=\frac{\rho}{1+\rho} .
$$

Theorem 2 implies the following statement.

Theorem 3. Stationary flows of the served customers and customers that have been rejected, are Poisson and have intensities $a_{1}=b_{1} \mu, a_{2}=b_{1} \lambda$, respectively. The stationary output flow in the system $M|M| 1 \mid 0$ is Poisson and its intensity $a=\lambda=a_{1}+a_{2}$.

Remark 2. The statement of Theorem 3 extends to queuing systems $M|M| n \mid N$ with a limited queue, a finite number of servers, as well as a system with a finite number of flows and a fairly general discipline of their service. In the latter case it is necessary to fulfil the ergodicity condition, which requires that the graph whose nodes are the states of the system, whose edges are transitions between states, satisfies the reachable condition of any node from any other node.

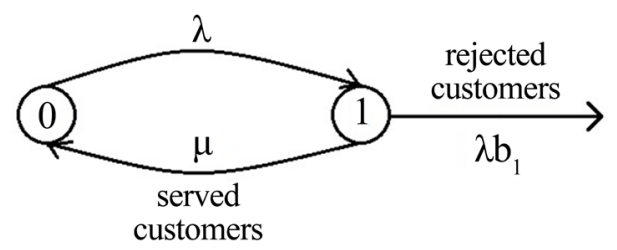

Figure 1. Transition intensities of process $z(t)$ describing the number of customers in the queuing system $M|M| 1 \mid 0$ with failures. 


\section{Queuing Systems in Random Environment}

1) Poisson input flow with randomly varying intensity. Let the time axis $t \geq 0$ be split into half-intervals

$$
\left[T_{0}=0, T_{1}=T_{0}+\xi_{1}\right),\left[T_{1}, T_{2}=T_{1}+\xi_{2}\right), \cdots,
$$

where $\xi_{1}, \xi_{2}, \cdots$ are independent random variables with distribution

$$
P\left(\xi_{k}>t\right)=\exp (-\sigma t), t \geq 0, k=1,2, \cdots
$$

with parameter $\sigma>0$. Introduce discrete Markov chain $n_{1}, n_{2}, \cdots$ with set of states $\{1, \cdots, M\}$ and irreducible transition matrix $\left\|\theta_{i, j}\right\|_{i, j=1}^{M}$ and stationary probabilities $\pi_{i}, i=1, \cdots, M$.

Consider the Markov random process $(i(t), x(t)), t \geq 0$, where $i(t)$ is a Markov stationary random process that characterizes the intensity of the input flow at the time $t, x(t)$ is the number of input flow customers that came to the system on the half-interval $(0, t]$. Example of this process is represented in Figure 2. Here $\alpha_{1}$ is an intensity of a transition from $(1, x)$ to $(2, x)$, and $\alpha_{2}$ is intensity of transition from $(2, x)$ to $(1, x)$, and $\lambda_{1}$ is intensity of transition from $(1, x)$ to $(1, x+1)$, and $\lambda_{2}$ is intensity of transition from $(2, x)$ to $(2, x+1), x=0,1, \cdots$.

Points of jumps (up) of the process $x(t), t \geq 0$ are the moments of customers arrivals to the system. Since the process $(i(t), x(t)), t \geq 0$ is Markov, then for a random flow defined by jumps (up) of the component $x(t)$ satisfies the condition a) of the Section 3. In turn, due to the stationarity of the Markov process $i(t)$ the probability occurrences of process $x(t)$ jump in the half-interval $(t, t+h]$ equals $\bar{\lambda} h+o(h)$, where $\bar{\lambda}=\sum_{i=1}^{M} \pi_{i} \lambda_{i}$. Thus, it is proved that the input Poisson flow with a randomly varying intensity coincides by the distribution with the Poisson flow having an average intensity $\bar{\lambda}$.

This circumstance allows us to study the output flows in queuing systems and networks with Poisson input flow having a randomly changing intensity, assuming that the random service intensities in the nodes of the queuing system or network and the random input flow are independent.

2) Queing system $M|M| 1 \mid 0$ in random environment. Consider the system $M|M| 1 \mid 0$ with the service intensity $\mu(t)$ and the Poisson input

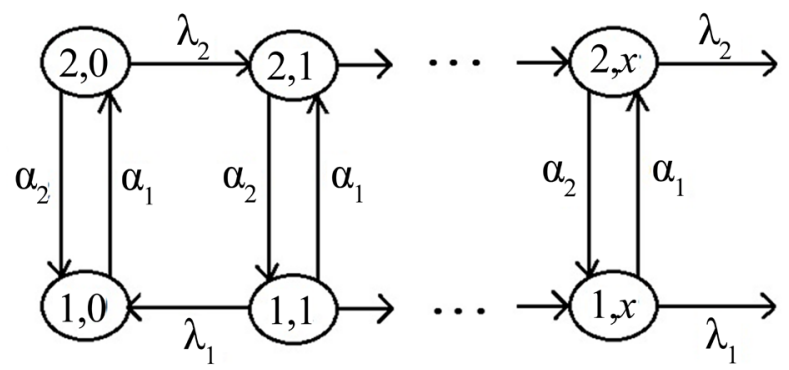

Figure 2. Transition intensities of process $(i(t), x(t))$ describing the number of input flow customers that came to the system on the half-interval $(0, t]$. 
flow with the intensity $\lambda(t)$, that change randomly according to the following rules. Let on each half-interval $\left[T_{k-1}, T_{k}\right)$ the input flow to the system $M|M| 1 \mid 0$ be Poisson with the intensity $\lambda(t)=\lambda_{n}, t \in\left[T_{l-1}, T_{l}\right), l=1,2, \cdots$, and the intensity of service is $\mu(t)=\mu_{n_{l}}, t \in\left[T_{l-1}, T_{l}\right), l=1,2, \cdots$. It is easy to check that under these conditions, the Markov random process $(i(t), x(t))$ is ergodic. Then from Theorem 2 it is easy to establish that the total output flow from a given system is Poisson with the intensity $\bar{\lambda}$. These constructions allow to consider manifold queuing models with different input flows and distributions of customers service times.

Partially supported by Russian Fund of Basic Researches, project 17-07-00177.

\section{References}

[1] Burke, P.J. (1956) The Output of a Queuing System. Operations Research, 4, 699-704. https://doi.org/10.1287/opre.4.6.699

[2] Tsitsiashvili, G.Sh. and Osipova M.A. (2018) Generalization and Extension of Burke Theorem. Reliability: Theory and Applications, 13, 59-62.

[3] Khinchin, A.Ya. (1963) Works on the Mathematical Queuing Theory. Physmatlit, Moscow. (In Russian)

[4] Borovkov, A.A. (1972) Course of Probability Theory. Nauka, Moscow. (In Russian)

[5] Ivchenko, G.I., Kashtanov, V.A. and Kovalenko, I.N. (1982) Queuing Theory. Vishaya Shkola, Moscow. (In Russian) 